\title{
Smart Home Applications for Energy Saving and Increased Security
}

\author{
Lambros Katsinoulas \\ Dept. of Automation Eng. \\ PUAS \\ Athens, Greece
}

\author{
Michail Papoutsidakis \\ Dept. of Automation Eng. \\ PUAS \\ Athens, Greece
}

\author{
Dimitrios Tseles \\ Dept. of Automation Eng. \\ PUAS \\ Athens, Greece
}

\begin{abstract}
Nowadays, because of the current conditions in our country and globally, in general buildings security and all installations is primary concern for all of us. Primary goal is also energy save, to which there has been given a lot of importance by industry, and not only, as a result of the financial crisis, but also for the protection of the environment. Energy save is an important part of research and implementation sector, which includes methods, specialized systems, informations systems, autonomous control systems, by which the consumed energy can be reduced. In this paper, the design and implementation of united alarm systems will be presented along with a light control system. The main idea is for these two systems to be united and installed in an imaginary company whose goal is energy save with least possible interventions. In this part there will be given more emphasis given on the simulation of the PLC program for educational reasons and better presentation of its function. Subsequently, the model's implementation process will be analyzed along with her results, which is going to give us the trigger to discuss the advantages and the disadvantages of the system. As a conclusion, possible future optimizations will be proposed as well as the possibility of the implementation in already installed systems. Aim of this thesis is to show the advantages of the systems interconnection, same time with systems ease of use as there is not any other similar system in the market
\end{abstract}

\section{Keywords}

Energy saving, Energy saving in lighting systems, Security systems, Alarm system, PLC

\section{INTRODUCTION}

Energy has been, is and will be the leading force of every human activity. The word energy is first found in 1959, but the actual meaning in physics is not clear until 1850. In 1807, Thomas Young was the first to use the term "energy" instead of "living force" to mention the product of the mass of a project multiplied with the square of its speed. In 1829 Gustave-Gaspard Coriolis described the term kinetic energy with its today meaning and in 1953 William Rankine came up with term "potential energy" [1]. The term "sustainable energy" includes a series of practices, policies and technologies by which we are trying to cover our energy needs with the least environmental, social and financial waste. That is achieved with the improvement of the energy efficiency of the process and the exploitation of the energy sources. Energy save is the improvement of the performance level in energy use. It is completed with the reduce of the consumption of primary forms of energy per unit of product and the reduce of the energy cost per unit of final product. A great energy consumer who has a high potential for energy save are the buildings. With the help of the right techniques and the use of efficient technologies, the achievement of great improvement of energy efficient at buildings with environmental and social benefits, is possible. The total amount of the electrical energy consumed from lighting, is also affected by the installation time, which is affected by the natural light, the use of space and the existence of the right light control systems manual or automatized, for the ensurance of the best visual comfort conditions, without the pointless function of the system.

\section{SECURITY SYSTEMS}

The term security systems refers to all the provisions and devices which by the right connections guard a place from malicious actions against privacy and property. An alarm system through its provisions, first of all targets a preventing any kind of space violation and if that doesn't seem to be enough, being able to inform us in time. Main parts of the circuit is the central unit or control panel, one or more keyboards, random interior or exteriors detectors, inside or outside sirens, random expansions interface boards for internet connection or another security system.

Control panel

The most import selection in an alarm system is this of the central unit, which undertakes to process the different signals that are being sent by the sensors of the system. The basic abilities that can affect the selection are the following.

- Number of inputs on the main board as well as the max possible number with the expansions boards.

- Ability of zone separation into groups. The modern units, allow the installer and the user to separate an alarm system into parts.

- Provided event memory, refers to the file that the panel keeps according the damages, problems, alarms as well as every user's actions.

- Extensions and interconnect devices. Refers to all the devices that give the alarm the possibility to connect with the internet, with other devices and systems.

Control units, detectors and alert devices

Control units refers to all those peripherals with which the system can be armed and disarmed. Some examples of these are keyboards, remote controls, rfid devices and remote control by use of phone or computer applications. Alarm systems detectors are the sensors that are connected with the control panel in order to detect any kind of space violation. They can separate in two big categories, supplied detectors and non-supplied detectors. Alarm alert devices refer to all provisions that provide sounder or light warning of alarm alert so that the owner and the neighbors to be informed for the 
violation. The sirens are separated in two categories according to their placement, indoor and outdoor sirens.

\section{Expansion boards}

The technological developments along with the modern facilities created the need of all these units that are connected with the main board through the serial communication bus. We can separate them in three categories, inputs and outputs extensions units and communication units. The continuous development in security systems has created addressable sensors which are also connected with the bus of the main board, offering many chances of parameterization through the programming software of the panel. The wireless sensor allows us the chance to extend the control panels with the use of wireless devices, allowing same time the installer to create o hybrid system composed by wired and wireless zones.

\section{PROGRAMMABle LOGIC CONTROLLER}

The main feature of an automation system is that it can run without human surveillance or intervention and run in a way that the desired result is achieved with the predetermined accuracy. The rapid development of technology and especially computing, directly affects all the modern automated functions of professional and household equipment. The first PLC appeared in the end of 60's in the USA to cover the needs of automation in the American car industry. Instead of wire connection and welding, the handling was going to be done through programming in the system, exactly like the computer. The production processes have become more complex, the dead times in production are continuously reduced and the needs for quality are increased. PLC is nothing but a micro-computer, suitably adapted so it can used for the automation function. It is a digital electronic system, designed for uses to industrial environment, which uses a controlled memory for the programming commands storage so that the different functions can be accomplished, like logical, time, metric and numerical operations and they can at the same time to control different machines and processes by analogue/ digital inputs. The main parts of a PLC is the CPU (Central Processing Unit), the Input-Output unit (I/O), the power supply and the programming unit.

\section{Advantages}

The PLC provide many advantages compared with the control by conventional relays. In case of industrial processes it probably is cheaper to change the whole control system than redoing the wiring part. The wiring is almost eliminated by the use of the PLC since the program takes over the whole logic of control. Furthermore during the implementation stage, changes can be easily done at any time, which at the classic automation would be extremely time consuming. The place of the control panel of a process has been dramatically reduced with the use of the PLC.

The user is being offered by the benefits in problem solving and the location of the damages by the use of programmable controller. By the help of the programming we can check the process of the performance and through diagnostics to locate any damages. The visual control of the function or nonelements of the installation is easily done with the help of the led that can be found in all plc expansions. Every change in the user's program is saved in the memory, in this way the technician isn't confused by non-up-to-date wiring diagrams.

The control of the operation can also be done remotely through industrial network. The system reliability is more than the ordinary automation, since the system do not involve moving parts. The result is no mechanical damages and zero maintenance demands. PLC are devices that can be used in many applications since are not closed systems. The user can extend the already used system by adding the appropriate I/O units, provided that prevision has been done in the stage of the system design. The potentials of system interconnection of PLC with computers can easily turned into the control of whole system from a specific location in minimum time along with database information in real accounting and warehouse timing [2].

\section{METHODOLOGY-UNIFIED SYSTEM}

This thesis was inspired by observations in existing buildings that had motion and presence sensors for different systems in same locations. It was also observed that there were alarm sensors as well as motion sensors for local lighting control. The question being raised is if the sensor could give the detection signal in both systems. The solution can be found through the automation by giving collaboration and integration system features. Aim is cost saving by reduce the number of the sensors in half. At the same time, an effect will be made for other potentials to be given in both systems to their interaction.

\subsection{Planning of the unification}

In the following plan view the place where the integration of the systems will be implemented is appeared. It consists of four offices and a hall, place where a small company can be established. In every office there will be o motion sensor as well as in the hall. The lights will be manually controlled by push buttons, while for the hall there will be two control points.

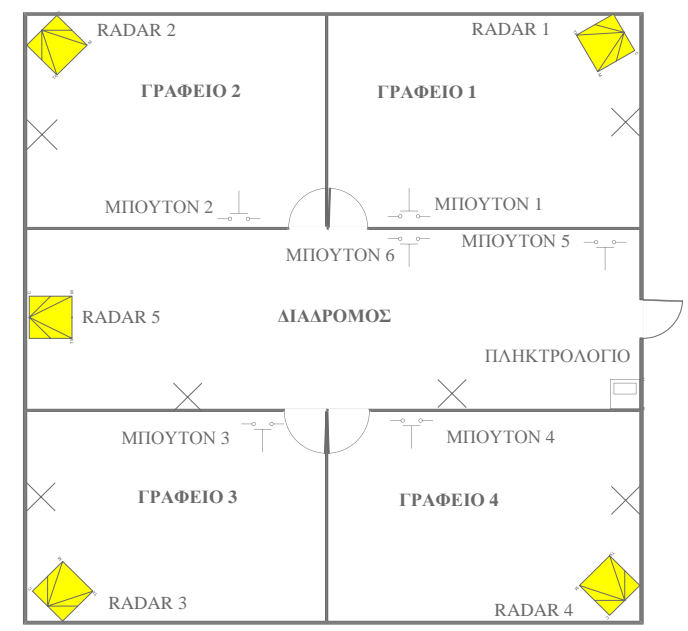

Fig. 1 Plan of the company

The user will be able to turn on and off the lights but through the PLC there will be a presence control. In case there is not motion for time $t$ there will go off the lights in the particular place. The electrical wiring of lights follows the same rules as control, where from each lamp or lamp array, the wires should end in PLC so they can connect with their outputs. The operating voltage that is going to be used in this thesis is of $12 \mathrm{~V} \mathrm{dc}$, if the lamps had voltage of $240 \mathrm{~V}$ ac, then the appropriate safety provisions should be used to protect the system and the users, according the ELOT HD 384. The powering of PLC will be given main alarm panel while the lightings by a different power supply. 


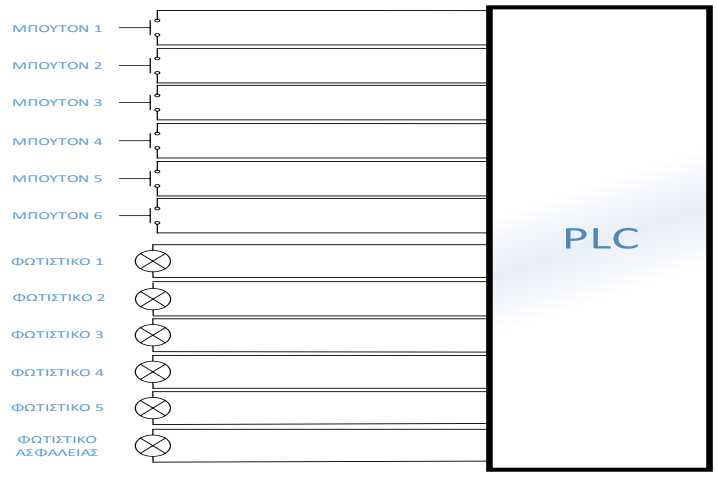

Fig. 2 PLC I/O

The PLC that was used in this thesis is Zelio SR3B261JD. It belongs in small programmable logic controllers Zelio logic of Schneider with operating voltage on $12 \mathrm{~V}$ dc. The choice was based on the alarm panel which can adequately power it with $12 \mathrm{~V}$. It has 16 inputs of which 6 are analogue, covering the needs of the application. The outputs are 10 with the potential of increase by an extension unit. The programming can be done through the LCD of Zelio or through a computer in FBD or Ladder language [3].

In the security system of the application the design might be considered in adequate since every space is covered by one sensor, but that is done in purpose so the interconnected sensors can be obvious. The chosen main panel for this thesis was Proteus (S-Pro 32) by Sigma security. The programming of the panel can be locally done by a keyboard but also by computer [4].

The wiring of the system will follow the instructions of the manufacturer of the main panel as well as the manufacturer of the sensors. Every sensor needs 2 pairs of wires, one is used for the power supply and the other for the zone, transferring the situation of the sensor to the main panel. The connection of the sensors will be done as shown below, by changing the zone that each sensor corresponds with starting from zone 1 to 5 .

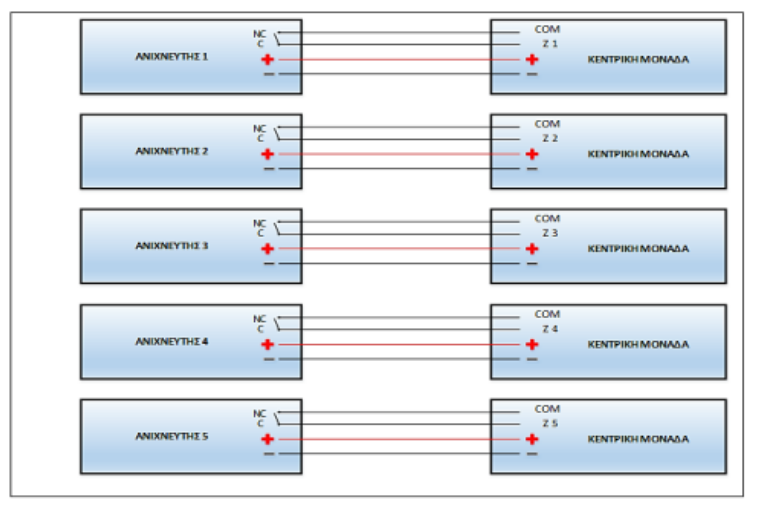

Fig. 3 Sensors wiring

There will also be a buzzer located in the alarm system so there be a sound alert in case of violation. The buzzer will follow the same connection with an indoor siren of bigger intensity. The (-) of the buzzer will be connected with the (-) of the board plugs that are intended for the supply of the sirens and the (+) in the SR plug of the main board.

The keyboard has its own connectors on the board since is a device that has two way communication by bus with the main board. It requires 3 wires for its proper function, two of which are used for the supply and one for bus [5].

The design of the security system will require one more device for the needs of the thesis which is an output expansion. The outputs needed for the PLC inform of motion detection are 5 one for each radar. One more output that is going to be needed in case of an alarm. In case of a violation, apart from the sound devices, all lights will be turned on, with ultimate goal of creating anxiety for the burglar, as well as a better video capture from a closed circuit system that can also exist. Another output will be used to inform the loss of $240 \mathrm{~V}$, so that an emergency light turn on in the hall. The supply of the specific lamp will be given by the alarm panel, which has a battery in case of power out. The connection of the expansion with the main board is the same with the keyboards since it's a unit that interacts with a bus [6].

The connection to the PLC can be done in two ways since the specific output board does not give us dry contact via relay, but open collector outputs. The first is to drive a relay from which outputs plugs are going to be used and the second is a direct connection with the PLC input according to the manufacturer's instructions. This type of outputs can only produce one of the logic levels, for the production of the second level the use of an external resistor is needed, which is connected to the corresponding power line. The preferred option is the second since there is no need for more materials and the complexity of the system is reduced [7].

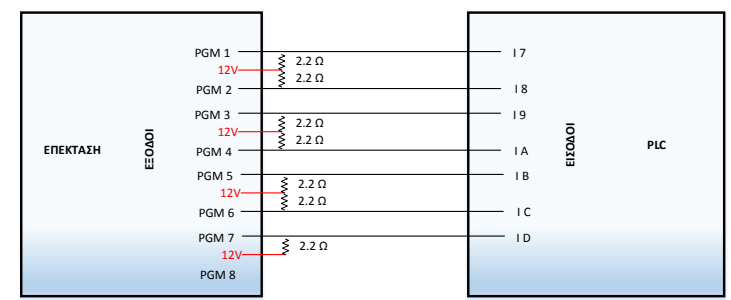

Fig. 4 PGM output wiring

\subsection{Security system programming}

The alarm panel's programming will be done through a computer. For the programming procedure there should be a program installed at the computer and that is Sload by Sigma Security which is indented for the S-pro series panels. The communication of the computer with the panel needs a connection module, the USB to mini USB LDL by Sigma.

The tab that is going to be used for the programming is the zone programming. This is one of the most important parts of the programming procedure since that is where the systems inputs are defined. The number of the zones that are going to be activated at the system is five, as many as the sensors in the offices. First choice in the zone characteristics is the zone type. The chosen type for the application is an IR/MWperimeter radar. The description of the zone is coming next, the given name be for an easy separation of the zones.

By moving the bar that is under the options towards right more fields are appeared. Through those fields we are going to adjust the zone's termination way, which is to be closed in state of inactivity and the hall's sensor entry time that will be 20 seconds. The only change between the factory programming is that the unused zones should affect the system. The only tabs we are going to use from the left ones, is the PGM programming and that is because communication 
with central station by telephone line, TCP-IP board or GPRS, is not going to be used.

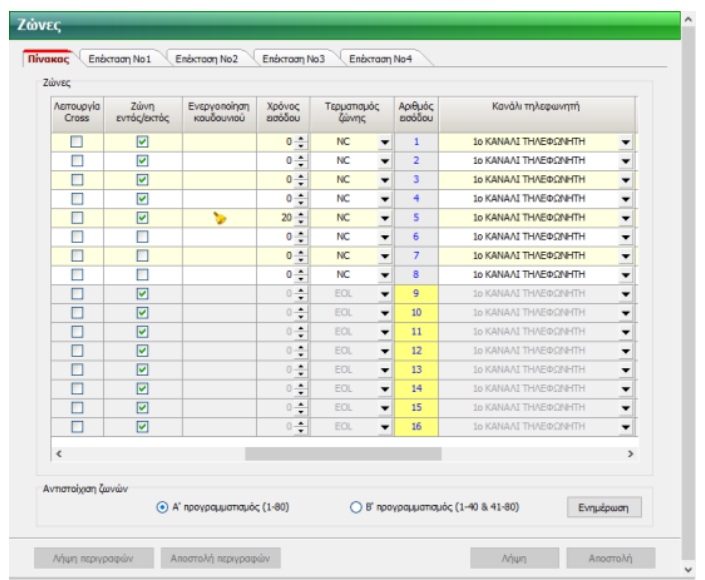

Fig. 5 Zone programming

The consolidated system's from the alarm, is to inform the PLC in case of motion detection and that is going to be achieved through the PGM. On the following picture, you can observe that the total of the programmed outputs rises in 18 . Output number 1 and 2 correspond to the main board's outputs while the rest to the expansions. According to the programming case an expansion output board is used, that will correspond to the number 11 to 18 outputs. Numbers 11 to 15 will correspond to the sensors. It is defined that he output in state of inactivity is going to be $0 \mathrm{~V}$ and is going to follow the state of zone with 1 minute time. The next output will be the one to inform the PLC in case of violation so that the whole lights will be activated. Its programming is to give $0 \mathrm{~V}$ in state of inactivity, follow the area 1 where the whole system is and remain in action follows alarm.

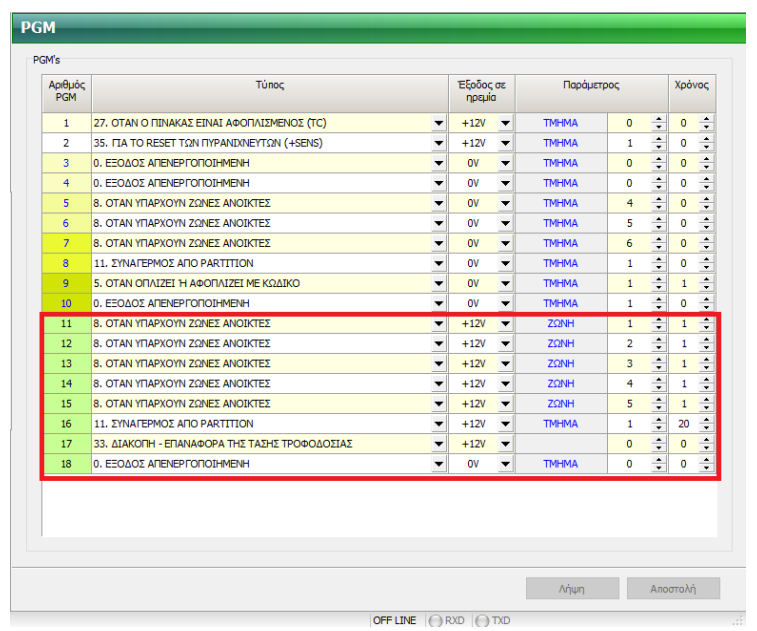

Fig. 6 PGM programming

Last output that is going to be used, is number 17 which will activate the emergency light through the PLC in case of power out on $240 \mathrm{~V}$ ac. The output is going to give $0 \mathrm{~V}$ in state of inactivity and the activation time will be 0 , since the emergency light on should be direct.

\subsection{PLC programming}

The PLC programming is going to be accomplished with the use of a computer. For the procedure of the programming, Zelio Soft 2 of Schneider should have been installed at the computer, which is intented for the programming of Zelio logic series. A local programming device, SR2 USB01 is also going to be needed, so that the computer can communicate with the PLC, as well as the installation of its driving programs at the computer. During the first inception of the program, a window will appear with the options of the creation of a new program, open an existing, download from unit and live unit monitoring. The choice will be a new program to be created. Subsequently, a dialog box will be created by the program, in order to help the installer choose the characteristics of the controller to be used. Through the icons, the application's PLC is being detected, alongside, the unit possibilities can be read in summary.

The PLC programming can now be done by defining the system's inputs and outputs at the first place. There are 6 control points of the lighting for 5 spaces, from where the user will be able to turn on and off the lights whenever he decides. Same time, through the sensors the PLC should be able to control if there is motion in the spaces for $t$ time, so when there is motion indeed, the time can be renewed. Until now, in our hypothesis there are 11 inputs, 6 from buttons and 5 from the sensors. Wanting to give a characteristic to the security system where in case of violation in one of the spaces, all lights will be on, one more input will be needed on the PLC. Finishing the requirements of inputs and outputs another input will be needed which is going to inform PLC in case of ac loss so that the emergency light can be activated in time. The total number of the outputs in use rises in 13 while the outputs in 6 .

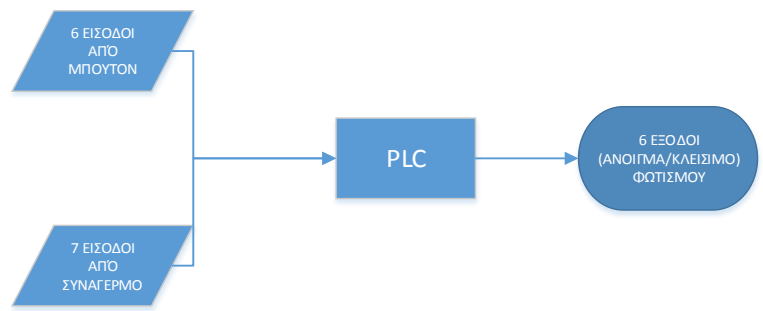

Fig. 7 Input-output diagram

The lighting's control point choice will be a push button with open contact in state of inactivity. For this to be achieved an impulse relay will be needed, where every time the button going to be pressed, its state will be changing along with the lights. Same time, another timer should be activated by passing of which, no motion will be detected the lights can go off. To close, in case of alarm, a contact should be exist, to activate every office's lights. On the following picture, the first office's program is presented.

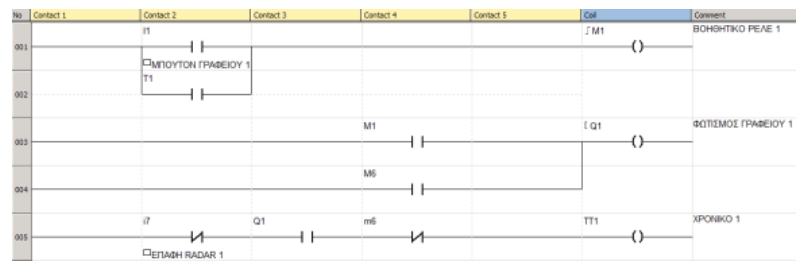

Fig. 8 Ladder diagram of office 1

By following the program's route, as soon as contact I1 is pressed, the auxiliary relay M1 will be activated which will act like an impulse relay. On line 3 relay's contact M1 will close by activating the lighting output. On line 5 , output relay's contact Q1 will close, in order for the timer 1 to be activated, so in time t the lights will go off. On the same line, there is also the contact that comes from i7 sensor's security 
system, which when opened, causes restarting to timer which means renewing the time t. By the time t passes, contact $\mathrm{T} 1$ will give a pulse, resulting the lights to go off, which actually mean pressing of the button so that M1 relay changes state. Contacts M6 and $\mathrm{m} 6$ are an alarms results. M6 directly activates Q1 relay that way lights turn on, while m6 doesn't allow timer to begin.

The logic is exactly the same for the rest of the offices. The only difference is at the part of the hallway where there are two control points. I5 and I6 buttons that correspond to the hallway, have been in parallel connection, this is possible since they cover the same space. So, in case more than one control point for the lighting exist, they can be connected in parallel locally, without the need for the wiring to reach the PLC.

\subsection{Simulation of a PLC program}

Having completed the programming part, the application allows the installer to control its proper function. The control process is done through simulation. By activating input I1 that corresponds to office's 1 push button line 3 and 5 become red. The activation is done by pressing the corresponding icon twice since the icon works as switch and not as button. For the project's needs, the following snapshots are on the first click, so that the motion and its corresponding input are appeared. As soon as, it is pushed the office's lights turn on and the timer 1 is directly activated.

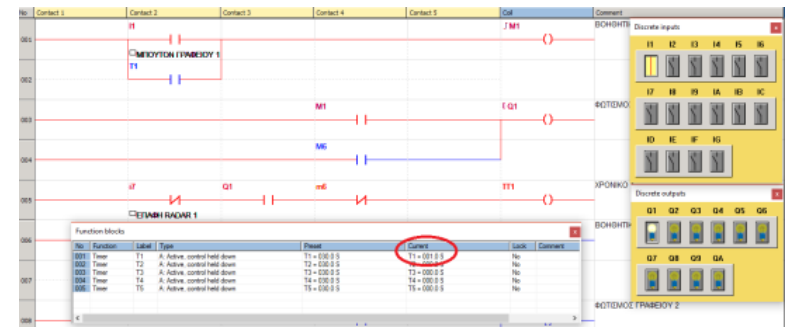

Fig. 9 Activation of contact I1

If input I1 reactivated, the lights automatically turn off but also the timer will stop working. If instead of the I1's activation, timer allowed to finish its circle, the result would be the same. Since after $30 \mathrm{sec}$ passing of time that has been defined, the timer simulates the clicking as it creates a pulse on contact T1. For this snapshot to be achieved, where the pulse is instant, the response time of the program has changed. It is clear that the auxiliary relay M1 activated by the contact T1.

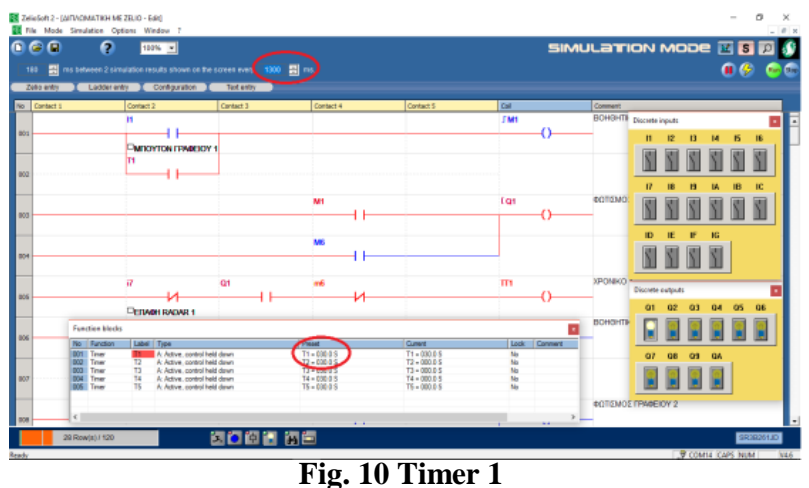

Next stage of the simulation is the control of the timer reset function by the motion sensing on the space. So by letting the lights on for office 1 , the contact i7 will be activated. It is observed that the lights are still on, but the timing is zero. From the snapshot, it is obvious that the timer is not powered on by voltage. The lighting control procedure through the simulation for the rest of the offices and hallway is the same.

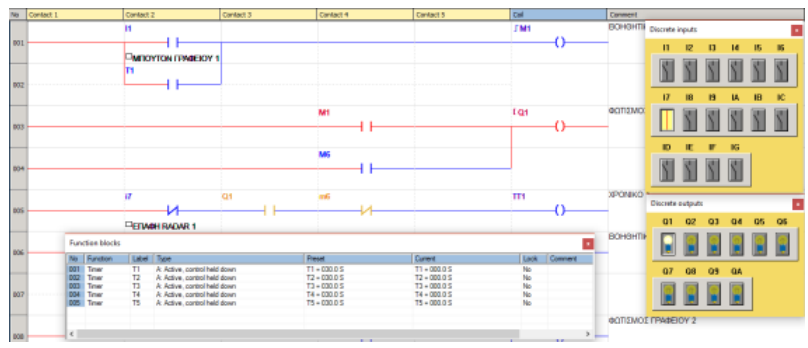

Fig. 11 Timer's 1 reset

In case of an alarm, input IC will be activated. The following result is for all power lines of the lighting to be activated, without the activation of the timer relays. The relays came out of the contacts $\mathrm{m} 6$ on their branches, since there is no reason for them to be activated, as the alarm time is defined by the security system.

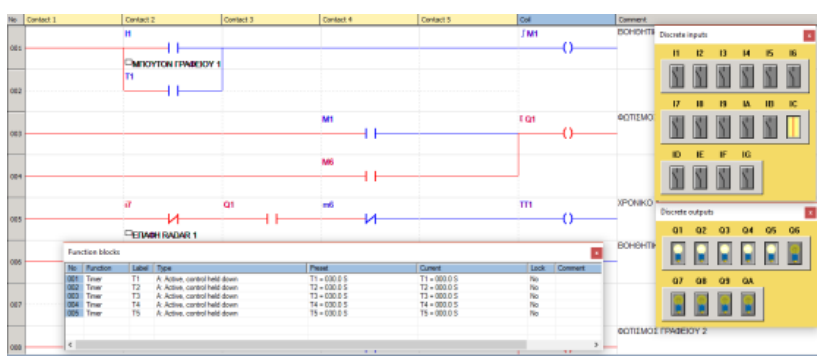

Fig. 12 Alarm activation

The final output that can be controlled is the output for the emergency light through the contact ID. Its activation is controlled by the alarm in case of a power out while its deactivation by the power on.

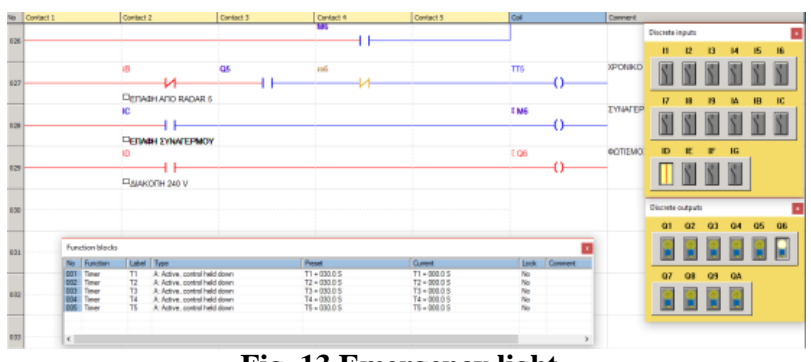

Fig. 13 Emergency light

\subsection{Maquette's implementation}

The first stage of the implementation is the creation of the wooden construction to which all the application's materials were placed. The construction was $80 \times 80 \times 44 \mathrm{~cm}$ with 5 distinct spaces at the inside. The spaces represent the offices and the company's hallway for which the system was designed. After that, the materials were placed in the inside places. These were the sensors, as well as the led films that illuminate each place, by completing their wirings same time. 


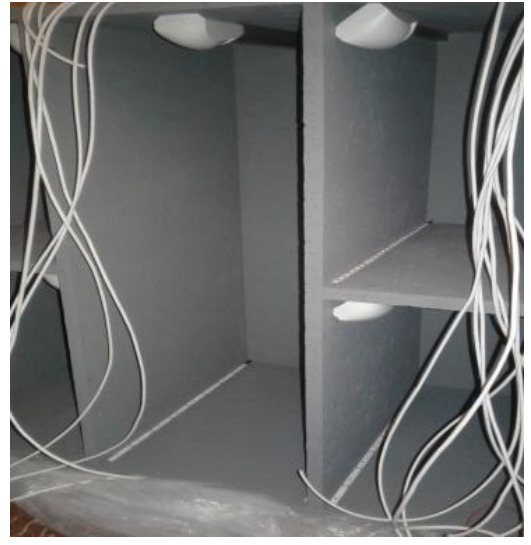

Fig. 14 Maquette's wiring

The implementation is completed with the terminations at the wirings and the connections on the main units.

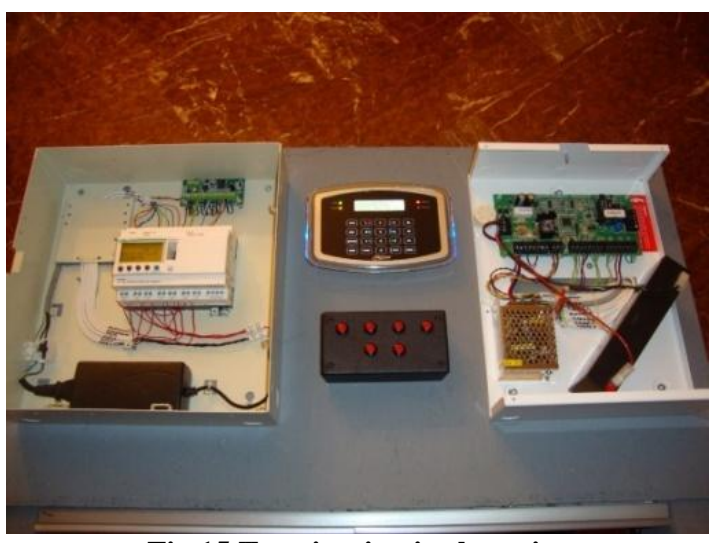

Fig.15 Termination in the units

After sending the ready programming from the computer to the systems, the operation controls of the systems were completed. On the picture below, the system in a stage of alarm is shown, with the lights activated in all spaces.

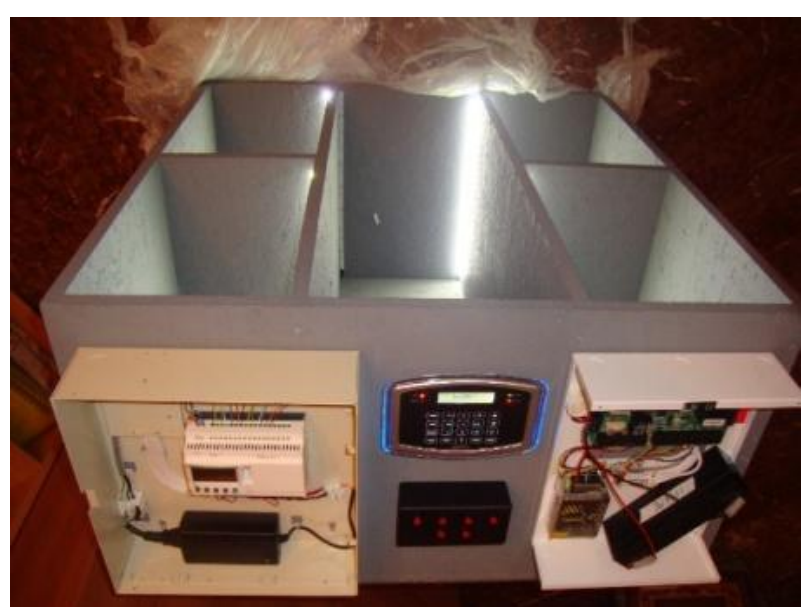

Fig. 16 Alarm activation

\section{CONCLUSIONS AND FURTHER PROSPECTS}

The completion of the implementation of the model give us the thesis's final result. Starting from the theoretical part of the research and the design, the system is transferred into real conditions, even if they are controlled. The basic aims set at the design stage were achieved. In detail:
1. The security system's sensors adequately provided the system with the control of the lighting by motion detection. PLC, through the programming can sense that there is not motion in the space and turn off the lights. The final result is energy save.

2. In case of space violation the security system is activated, which after that informs PLC that activates the whole lighting. In this case, the lighting circuit works as output device of the alarm.

3. In case of power cut off by the provider, the alarm is going to keep working with the help of the backup battery by powering the same time PLC with the result for the emergency light to turn on.

4. Through the programmed controller, automation in the light control is achieved, in the first case user controls the lighting manually. In the second case, the process has been automated since the control is now achieved through a microprocessor by giving the chance of manual control as well.

\subsection{Restrictions of the united system}

- First set restriction on the system, is his size. For a two offices expansion an expansion board should be installed at the PLC since two extras inputs from control push buttons and two inputs from the sensors of the security system are also going to be needed.

- Another restriction is the place where the installation is going to be done. In old installations, which use classic light wiring methods, where the light switch cuts off the active conductor of the circuit, cannot be implemented, since the cost of the interventions can be deterrent. The united system can be applied to old electrical installations, as long as the lighting wirings have the form of the bellow shape [8].

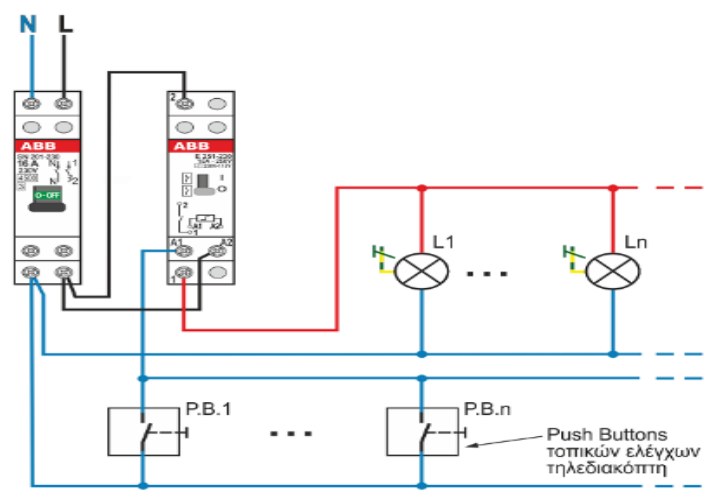

Fig. 17 Light wiring with impulse relay

\section{$5.2 \quad$ Future prospects}

The systems development can follow two directions the remote control of the application through the alarm or the PLC and the replacement of the PLC with a microprocessor so it can be joined to the Internet Of Things. The first option is to use the GSM of Schneider which will give to the application the opportunity of remote control through mobile phone. The operation can be done by text messaging. The second option for remote control can be created by adding ip-module of Sigma to the system. The Ixion apart of the security system control, can also control the system outputs. So by using a few outputs the right triggers can be given to the PLC with the 
result of the lighting control. The plethora of interconnection and control capabilities create totally personal applications according to the final user's requirements.

Place Tables/Figures/Images in text as close to the reference as possible (see Figure 1). It may extend across both columns to a maximum width of $17.78 \mathrm{~cm}$ (7").

Captions should be Times New Roman 9-point bold. They should be numbered (e.g., "Table 1" or "Figure 2"), please note that the word for Table and Figure are spelled out. Figure's captions should be centered beneath the image or picture, and Table captions should be centered above the table body.

\section{ACKNOWLEDGMENTS}

All authors would like to express their gratitude to the Piraeus University of Applied Sciences for providing the required data and funding in order to undertake and complete this research project as part of "Industrial Automation" Postgraduate Program of Studies.

\section{REFERENCES}

[1] Energy and environment, Teaching notes, N.Andritsos

[2] Industrial electronics, Frank. D. Petruzella, Tziola Publications

[3] Operating manual Zelio Logic

[4] Operating manual Proteus

[5] Installation manual S-pro 32

[6] Installation manual Pgm-08s

[7] Elmo Control, App. Note: Open collector outputs

[8] Basic principles of lighting control applications, Training center ISC Schneider Electric 